\title{
Review of alternative access in transcatheter aortic valve replacement
}

\author{
Adam Banks ${ }^{1}$, Jeff Gaca $^{2}$, Todd Kiefer ${ }^{1}$ \\ ${ }^{1}$ Divisions of Cardiology, ${ }^{2}$ Cardiac Surgery, Duke University Medical Center, Durham, North Carolina, USA \\ Contributions: (I) Conception and design: All authors; (II) Administrative support: None; (III) Provision of study materials or patients: None; (IV) \\ Collection and assembly of data: All authors; (V) Data analysis and interpretation: All authors; (VI) Manuscript writing: All authors; (VII) Final \\ approval of manuscript: All authors. \\ Correspondence to: Todd Kiefer, MD. Division of Cardiology, Duke University Medical Center, Durham, NC, USA. Email: todd.kiefer@duke.edu.
}

\begin{abstract}
Transcatheter aortic valve replacement (TAVR) has surpassed surgical aortic valve replacement (SAVR) as the most common treatment strategy for severe symptomatic aortic stenosis over the past decade. As TAVR technology has continued to advance, it has been expanded from being an option only for extreme risk patients to now being the preferred option for the majority of patients with severe aortic stenosis. Recent trials have shown that TAVR is superior or non-inferior to SAVR even in patients at low surgical risk. One limitation of TAVR is the need for large bore vascular access. This has improved over time with smaller sheath sizes and improved delivery systems, but is still a significant issue in a patient population that often has many comorbidities including peripheral arterial disease. Early in the TAVR experience the only option for alternative access was transapical access, which has consistently been linked to increased procedural complications and worsened clinical outcomes. However, in recent years several centers have demonstrated the safety and efficacy of several alternative access strategies including transaxillary, transcarotid, transcaval, and direct aortic. There are no randomized data comparing these strategies, so access site approach is chosen by the multidisciplinary heart team based on patient anatomy and site expertise. We will review the current data in alternative access that in our view supports prioritizing a transaxillary or transcarotid strategy. In addition, we will describe our center's pre-procedural planning, peri-procedural approach, and propose an algorithm for alternative access.
\end{abstract}

Keywords: Transcatheter aortic valve replacement (TAVR); vascular access

Submitted Jul 16, 2019. Accepted for publication Sep 29, 2019.

doi: $10.21037 /$ cdt.2019.10.01

View this article at: http://dx.doi.org/10.21037/cdt.2019.10.01

\section{Introduction}

Aortic stenosis is a progressive condition with a poor prognosis and no medical treatment options and ultimately requires valve replacement. Over the past decade, the development of transcatheter aortic valve replacement (TAVR) has provided an option for patients at high- and extreme-risk for surgical aortic valve replacement (SAVR). TAVR has also been approved by the Food and Drug Administration (FDA) in the intermediate risk population based on favorable clinical trial data, and recently, TAVR has been shown to be superior or non-inferior to SAVR even in patients at low risk for SAVR $(1,2)$. TAVR volume now exceeds the volume of SAVR in the treatment of aortic stenosis in the United States (3). The majority of TAVR procedures (92 percent) in the Unites States are performed via the preferred transfemoral approach (4). In addition, the majority of patients included in the Medtronic Corevalve and Edwards Sapien high and intermediate risk trials received TAVR via a transfemoral approach (5-8). and alternative access cases were excluded in the low risk trials $(1,2)$. However, approximately $25 \%$ of patients undergoing TAVR have significant peripheral arterial 
disease which has been linked to increased procedural complications and worsened clinical outcomes at 1-year (9). With this in mind, pre-procedural planning with gated cardiac computed tomography (CT) is essential to select an appropriate access site prior to the procedure. Early in the TAVR experience, the only alternative access was transapical access, which has consistently been linked to increased morbidity and procedural complications (10-13). Over the past several years several centers have published data demonstrating the safety of alternative access through transaxillary, transcarotid, transcaval, and direct aortic access. These approaches have now supplanted apical access. In 2013 , transapical access peaked occurring in over $40 \%$ of the TAVR cases in the United States (14). However, with smaller delivery sheaths and availability of alternative approaches the rate of transapical access or direct aortic access in the United States was only $4.8 \%$ from 2015 to 2017 and has continued to decline $(4,14)$. In 2017 , axillary access surpassed apical or direct aortic access as the most common alternative access strategy in the United States, although there is still limited utilization of carotid access (14). This review will describe the available data on alternative access and our approach to pre-procedural planning, access site selection, and periprocedural techniques.

\section{Approach to selecting access site}

Peripheral arterial disease is common in patients undergoing TAVR and vascular anatomy should clearly be defined prior to the procedure. TAVR patients should routinely have a high quality contrasted, arterial phase, gated TAVR-CT performed of the vascular anatomy extending from the mid neck to below the femoral head to include the common carotid, subclavian, aortic, iliac, and femoral arterial anatomy. Left or right femoral access will likely be appropriate in the majority of individuals, but in approximately $10-15 \%$ of patients an alternative access strategy will be required. We in general consider alternative access in patients with iliofemoral minimum lumen diameter (MLD) less than $5 \mathrm{~mm}$, severe tortuosity or calcification, chronic arterial dissection/thrombus, morbid obesity (body mass index greater than 45), or severe abdominal aortic atherosclerosis. These patients should be considered for alternative access preferably through the axillary or carotid artery.

Early in the TAVR experience, the only available access strategies were transfemoral or transapical. Transapical access has consistently been linked to increased procedural complication and increased morbidity and mortality and is of primarily historical significance in TAVR at this point (10-13). The Medtronic Corevalve high and intermediate risk trials allowed axillary or direct aortic access. However, only $17.2 \%$ and $6.3 \%$ of patients in the high and intermediate risk Corevalve trials received TAVR via an alternative access strategy $(5,6)$. In addition, the Partner A and B trials included transapical access and transapical or direct aortic access in $30.0 \%$ and $23.7 \%$ of the patients enrolled, respectively $(7,8)$. Given the limited enrollment of alternative access cases in these landmark randomized trials there was minimal data to guide clinicians on the choice or safety of alternative access in patients not suitable for transfemoral access. However, several observational retrospective studies have been published over the past several years that demonstrate the safety, efficacy, and feasibility of carotid and axillary access $(10,12,13,15)$. These studies have demonstrated high rates of procedural success with relatively low rates of stroke, major bleeding, and mortality. Of note a study from the transcatheter valve therapeutics (TVT) database comparing transaxillary $v s$. direct aortic or apical access was associated with a higher incidence of stroke with the axillary strategy (6.3\% vs. 3.1\%), but decreased all-cause mortality (13). In comparison, a study of transcarotid access from a French registry had a stroke rate of $1.6 \%$ which is comparable to stroke rates in transfemoral TAVR (15). These studies were in different cohorts of patients so cannot be used for direct comparison, but highlight the need for randomized data directly comparing an axillary versus carotid approach. These individual studies will be discussed in more detail below for the individual access sites. Given the lack of randomized studies comparing access approach at this point, alternative access strategy should be based on patient anatomy and heart center expertise, while selecting the least invasive approach that minimizes procedural complications and recovery time. A recent study from the TVT registry demonstrated that transfemoral access is being performed in greater than 90 percent of TAVR cases in the United States. As a result, need for alternative access is relatively rare. In this cohort, low volume centers had statistically higher rates of mortality for TAVR and this signal was increased in patients undergoing alternative access (4). Given that most low volume centers have limited experience with alternative access these patients should be referred to high volume TAVR centers that have extensive experience in multiple alternative access strategies.

Figure 1 shows our algorithm for alternative access at 


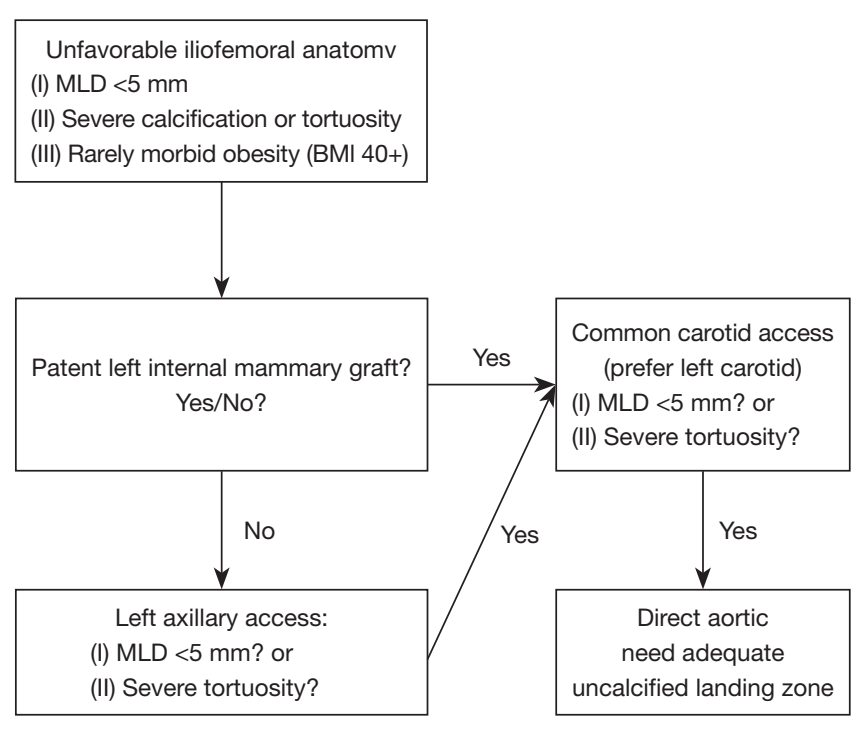

Figure 1 Duke Heart Center algorithm for alternative access. MLD, minimum lumen diameter.

Duke University Hospital. At our center, we use the left axillary artery as our preferred alternative access followed by the left or right carotid artery. The right axillary artery can be used, but provides a difficult angle for valve delivery so is not preferred. If the patient does not have suitable femoral, carotid, or axillary anatomy we then precede with a direct aortic approach. Using this algorithm, we have only utilized direct aortic access in four patients from 2014 to 2017 and have not turned down any patients for TAVR based on vascular access alone. Two of these patients received off-pump CABG at the time of their TAVR via direct aortic access. Our last apical access case was in 2015. Figure 2 displays our access trends from 2012 to 2017. For the past several years approximately $90 \%$ of cases have been completed through a transfemoral approach and transaxillary or transcarotid have now become our most common alternative access strategies. These trends are similar to national trends from the TVT registry.

\section{Axillary access}

In the United States transaxillary access is currently the preferred alternative access strategy when transfemoral access is not feasible. A recent retrospective study of TVT database demonstrated that over the past several years in the Unites States that there has been a rapid growth in the use of a transaxillary strategy, while the use of transaortic or transapical strategies have decreased. In this study, the device success rate for transaxillary was greater than 97 percent and after propensity matching axillary access was associated with lower 30-day mortality, shorter intensive care unit and length of hospital stay. However, there was a statistically significant increase in the stroke rate in the transaxillary access arm as compared to the direct aortic or apical arm $(6.3 \%$ vs. $3.1 \%)$ which warrants further investigation (13). This study helped confirm that a transaxillary approach should be preferred over a more invasive transaortic or transapical approach. Future studies are needed to directly compare a transcarotid versus transaxillary approach.

\section{Pre-procedural planning}

At our center, the current first alternative access strategy is a left axillary approach if the iliofemoral anatomy on the TAVR-CT precludes transfemoral access. The reconstructed images from the TAVR-CT are routinely reviewed through TeraRecon at our multidisciplinary conference that includes representatives from interventional cardiology, cardiothoracic surgery, and radiology. If the degree of iliofemoral atherosclerosis or tortuosity preclude transfemoral TAVR, we evaluate the axillary and carotids for potential alternative access. The left axillary artery provides a better angle of valve delivery given the natural curvature of the ascending aorta and is preferred over the right axillary artery. The MLD of the axillary artery needs to be at least $5 \mathrm{~mm}$ in diameter to safely deliver a sheath for TAVR. Figure 3 shows TAVR-CT images of a patient with suitable left axillary access with an MLD of greater than $6 \mathrm{~mm}$ and corresponding iliofemoral images with inadequate femoral access. A patent left internal mammary artery (LIMA) graft is not an absolute contraindication to a left axillary approach. However, if a patent LIMA graft is present, we prefer a transcarotid approach to avoid ischemia during the procedure or potential injury to the left axillary artery impacting flow to a patent LIMA. If a left axillary approach is chosen in the setting of a patent LIMA graft, the MLD of the axillary and subclavian should be at least 6 $\mathrm{mm}$. If the size of the axillary or subclavian is inadequate, we consider a carotid approach as our next strategy. For axillary access, we generally prefer the Medtronic Corevalve Evolut R device as the 14-French inline delivery catheter allows the whole system to be directly inserted into the subclavian artery. Although it is not our preference, the Edwards SAPIEN 3 can also be utilized for transaxillary TAVR. However, use of the SAPIEN 3 valve may be 
A

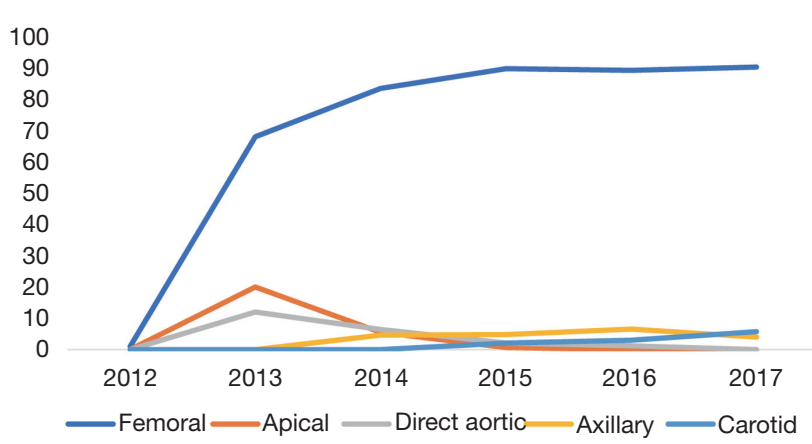

B

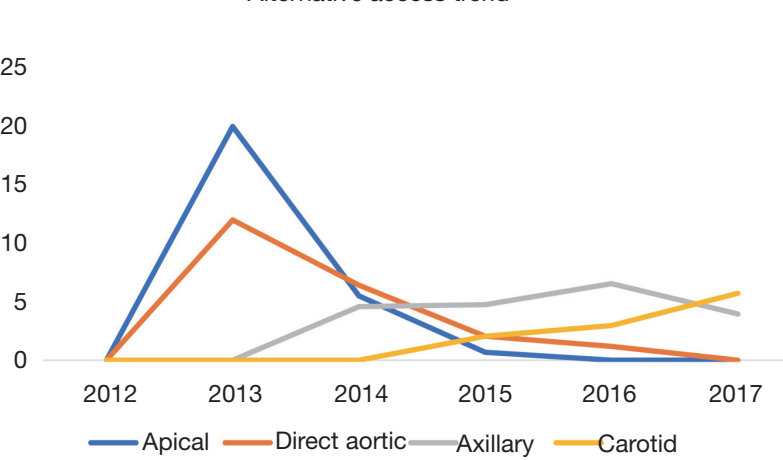

Figure 2 Access strategies for TAVR at Duke 2012 to 2017. (A) Percentage of access at Duke from 2012 to 2017 demonstrating 90 percent of procedures transfemoral from 2015 to 2017; (B) percentage of alternative access from 2012 to 2017 demonstrating axillary and carotid access replacing direct aortic and apical access. TAVR, transcatheter aortic valve replacement.
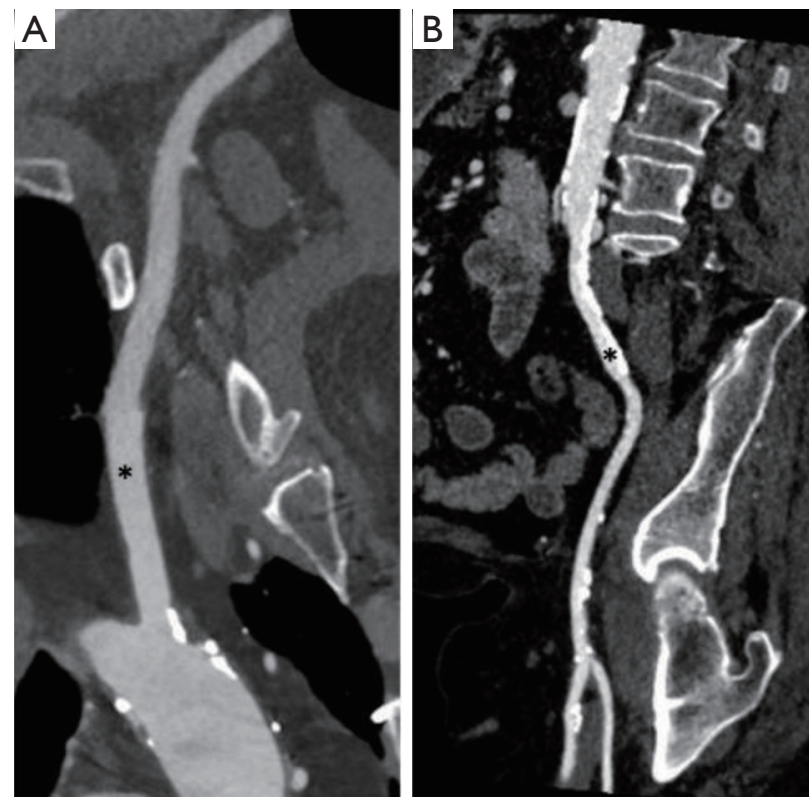

Figure 3 TAVR-CT in a patient that underwent left axillary access. (A) CT reconstruction from TeraRecon displaying suitable left axillary artery anatomy (marked by *) with MLD of greater than $6 \mathrm{~mm}$; (B) reconstruction from TeraRecon displaying iliofemoral anatomy with extensive calcified stenosis $\left(^{*}\right)$ and atherosclerosis predominantly in common iliac artery. TAVR, transcatheter aortic valve replacement; MLD, minimum lumen diameter; CT, computed tomography.

complicated by several factors: (I) extreme tortuosity of the axillary artery may kink the Edwards expandable sheath and render valve deployment impossible, (II) loading the valve on the balloon may be difficult due to a lack sufficient length in the ascending aorta and subclavian artery, (III) use of the Certitude transapical sheath (to allow the SAPIEN 3 to be loaded on the delivery balloon outside the body) requires larger axillary artery dimensions to accommodate the 18-21 French delivery systems.

Prior to the procedure, the patient is positioned supine on the table and the cardiac anesthesia team administers general anesthesia with endotracheal intubation. We favor general anesthesia over conscious sedation in alternative access cases for patient comfort during the cutdown. We generally prefer using open surgical exposure of the axillary artery over a percutaneous approach to minimize bleeding at the site and avoid potential brachial plexus injury. Although we have some experience with percutaneous axillary access for TAVR and several centers have also reported promising results, open axillary exposure remains the most common technique in our center (16). After the patient is intubated and sedated bilateral radial arterial lines are placed. The radial arterial line on the ipsilateral side of access is used to assess intact perfusion during and at the end of the case and the contralateral arterial line is utilized to assess blood pressure throughout the case as the valve deployment sheath often dampens the waveform in the ipsilateral arterial line.

After patient preparation is complete by cardiac anesthesia, a 4 to $5 \mathrm{~cm}$ transverse incision is made 1-2 finger breadths below the clavicle in the deltopectoral groove (Figure 4). Dissection is carried down to the pectoralis major fascia and divided with electrocautery. The clavipectoral fascia is then divided as the deltopectoreal 

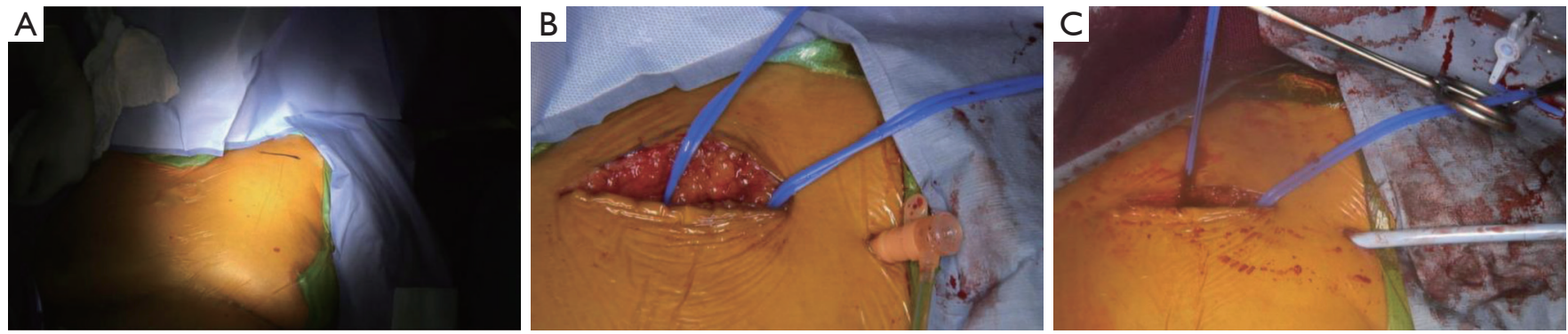

Figure 4 Left axillary artery cutdown technique. (A) Marked incision site 1-2 finger breadths below the lateral left clavicle; (B) cutdown with isolation of superior and inferior aspect of landing zone of left axillary artery after identifying brachial plexus. 7-French sheath inserted at separate site lateral to cutdown; (C) 7-French sheath exchanged for 14-French sheath.

groove is entered and the pectoralis minor is divided with cautery. The left axillary artery is then identified, and the brachial plexus typically lies superior to this with the axillary vein located inferior to the artery. The artery is dissected free over several centimeters while small branches from the axillary artery may need to be ligated with 2-0 silk ties. The artery can be accessed directly or through a separate $1 \mathrm{~cm}$ stab incision made lateral to the transverse axillary incision. Next, an access needle is inserted into the left axillary artery and a 7-French sheath is inserted via the Seldinger technique under direct visualization (Figure 4). The common femoral artery and common femoral vein are then accessed for placement the pigtail catheter in the sinus of Valsalva and a transvenous pacemaker in the right ventricle.

Once access is obtained the pigtail catheter is positioned in the non-coronary sinus (Corevalve) or the right coronary sinus (SAPIEN 3) from the femoral arterial access site and the c-arm is positioned in the optimal implant angle. An AL- 1 catheter is then used to cross the aortic valve and an exchanged for a pigtail catheter once the aortic valve is crossed. After hemodynamics (LVEDP and baseline aortic valve gradient) are recorded a deployment working wire, either a Medtronic Confida (Edwards Sapien) or a Boston Scientific Safari S2 (Medtronic Corevalve) wire, is inserted through the pigtail catheter into the left ventricle and the sheath is removed over the wire while maintaining digital pressure on the arteriotomy site. If pre-implantation balloon valvuloplasty is going to be performed, it can be performed through a 14-French Gore Dry Seal sheath or the Edwards sheath. After the valvuloplasty, the 14-French Gore Dry seal sheath can be exchanged for a 14-French inline Core Valve Evolut sheath for valve deployment. If the MLD is greater than $6 \mathrm{~mm}$ an 18-French Gore Dry seal sheath can be used for the entire procedure. Of note for both methods the tip of the sheath should only be advanced to the origin of the left subclavian to avoid interfering with valve deployment.

Valve deployment details are outside the scope of the current review, but it is similar to a femoral approach. Once the valve is deployed the delivery system is then removed over a working wire and a 14-French Gore Dry Seal sheath is inserted for hemostasis. The artery is controlled proximally and distally with clamps and the arteriotomy site is then repaired with multiple interrupted 5-0 Prolene sutures. When the repair is complete the clamps are removed, and hemostasis and distal perfusion are carefully assessed. The presence of an ipsilateral radial arterial line is especially helpful to confirm adequate distal perfusion and the integrity of the arterial repair. If hemostasis and perfusion are intact protamine is administered and the axillary incision is closed in layers. We do not routinely perform completion angiography of the axillary artery if we have confirmed adequate flow by the above techniques.

\section{Transcarotid access}

Transcarotid access emerged in the United States in 2013 and at select centers has increased in use as an alternative vascular access site depending on the heart center's expertise. However, transcarotid access, as of yet, has not had widespread adoption throughout the United States. Several registries have demonstrated that transcarotid TAVR has high rates of success with lower rates of complications compared to a direct aortic or an apical approach $(10,12)$. In our experience, prior carotid endarterectomy (CEA) is not a contraindication to carotid artery access for TAVR as the TAVR access site is below the prior CEA site. At the inception of transcarotid access there was understandably a fear that there would be a high stroke rate related to access 

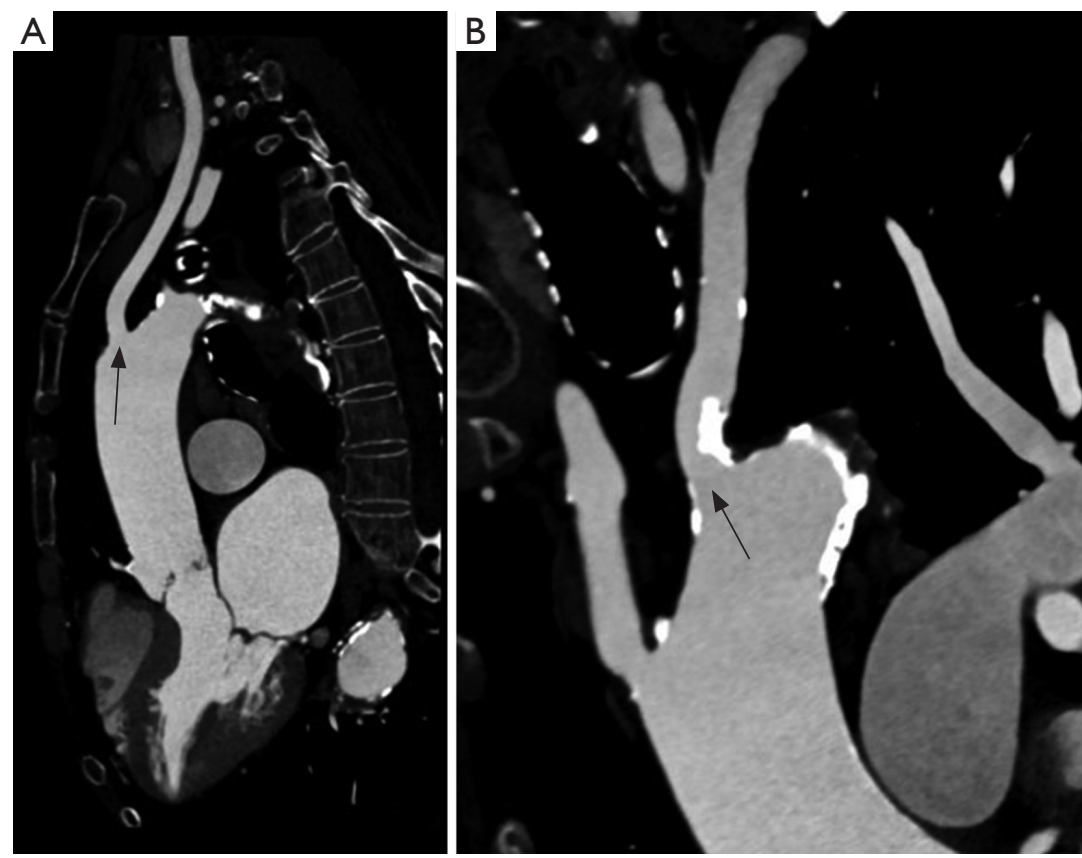

Figure 5 TAVR-CT in a patient that underwent left carotid access. (A) Left common carotid artery (arrow) with suitable anatomy with MLD greater than $7 \mathrm{~mm}$; (B) left axillary (arrow) with significant calcified ostial stenosis that is unsuitable for a left axillary approach. TAVR, transcatheter aortic valve replacement; MLD, minimum lumen diameter; CT, computed tomography.

site complications. However, registry data suggests that this has not been supported by observational data. A multicenter French Registry contained 314 patients that underwent transcarotid TAVR using the Edwards Sapien 3 device. In this cohort of patients, device deployment was successful in $97 \%$ of patients with low rates of major bleeding $(4.1 \%)$, new permanent pacemaker $(16.1 \%)$, and stroke or TIA $(1.6 \%)$ at 30 days (15). The stroke rate in this registry is similar to that of transfemoral TAVR and lower than what has been reported for transaxillary TAVR. This data supports that carotid access should be a preferred approach over a direct aortic or apical strategy. More data comparing axillary to carotid access in order to inform which access site should be the preferred alternative access is needed.

\section{Pre-procedural planning}

If the iliofemoral and left axillary arteries are not suitable for vascular access or if an intact left internal mammary graft is present, we evaluate and size the bilateral common carotid arteries for alternative access on gated TAVR-CT with the TeraRecon software. We prefer the left common carotid approach as the angle for valve delivery is preferable to the right carotid. However, the right carotid can be utilized and has a preferable delivery angle to a right axillary approach. The common carotid needs a MLD of $5 \mathrm{~mm}$ to accommodate the smallest sheath option (14 Fr inline Medtronic Corevalve Evolut-R) for carotid access, while the Edwards Certitude sheath (18 Fr for the 23 or $26 \mathrm{~mm}$ valves or $21 \mathrm{Fr}$ for the $29 \mathrm{~mm} \mathrm{~S} 3$ valve) requires a larger vessel with minimum MLD of greater than $7 \mathrm{~mm}$ for the smaller sheath size. Some centers have routinely performed CT or magnetic resonance angiography of the head and neck vessels to demonstrate an intact Circle of Willis prior to preceding with transcarotid TAVR. To date, there is no evidence that imaging the Circle of Willis is beneficial in planning transcarotid TAVR and we do not routinely perform intra-cranial vascular imaging prior to transcarotid TAVR. Figure 5 shows TAVR-CT images of a patient with an ostial left axillary calcified stenosis, but with a widely patient left common carotid with MLD of greater than $7 \mathrm{~mm}$.

\section{Procedure}

The patient is positioned supine on the table and put under general anesthesia with a radial arterial line for blood pressure monitoring. As stated in the transaxillary section, the patients undergoing alternative access are placed under 

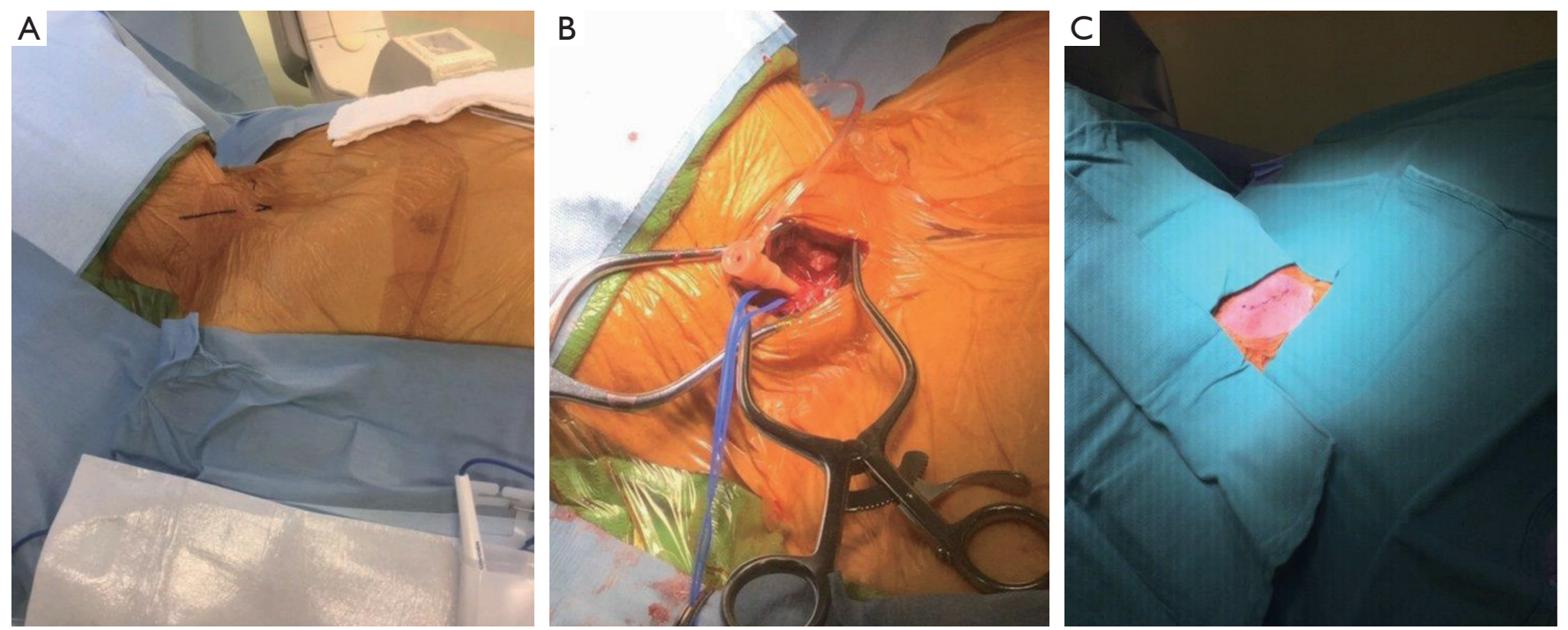

Figure 6 Right carotid artery cutdown technique. (A) Marked incision site for right carotid artery access; (B) cutdown with isolation of the right carotid artery; 7-French sheath inserted into the right carotid artery; (C) final carotid access site image after repair of the right carotid artery and skin closure.

general anesthesia rather than conscious sedation. After the patient is prepped and draped a $4 \mathrm{~cm}$ incision is made at the level of the sternocleidomastoid muscle just above the sternal notch (Figure 6). Dissection is carried out down to the level of the common carotid artery and the artery is dissected free over several centimeters. An access needle is then inserted into the common carotid artery and a 7-French sheath is inserted via the Seldinger technique (Figure 6). The common femoral artery and common femoral vein are then accessed for the pigtail catheter and transvenous pacer. If there are issues with femoral venous access, the 6-French pacing sheath may be placed in the internal jugular vein under direct visualization.

Once access is obtained the pigtail catheter is positioned in the appropriate coronary sinus from the femoral arterial access site and the c-arm is positioned in the optimal implant angle. An AL-1 or short Berenstein catheter is then used to direct a soft-tipped straight wire to cross the aortic valve and an exchanged for a pigtail catheter once the aortic valve is crossed. After hemodynamics are recorded, the working wire is inserted through the pigtail catheter into the left ventricle and the sheath is removed over the wire. The 7-French Sheath is then exchanged for an Edwards Certitude sheath or a 14-French inline Medtronic Corevalve Evolut-R delivery system depending on which valve is being used. After the valve is deployed the sheath is removed and the common carotid artery is clamped proximally and distally and this is repaired with multiple interrupted 5-0 prolene sutures. Care is taken to appropriately remove air from the carotid artery before the distal clamp is removed. The common carotid artery is then examined for hemostasis and pulse and then protamine is administered, and the carotid incision is closed. Again, we do not advocate the performance of completion carotid angiography in this access location.

\section{Direct aortic access}

Direct aortic access through a mini-sternotomy or suprasternal access approach can be used for TAVR if there is unsuitable femoral, axillary, and carotid anatomy. Retrospective analyses have shown that axillary or carotid access has lower rates of major bleeding and mortality as compared to a direct aortic approach. However, these same analyses have demonstrated that a direct aortic approach for TAVR trends towards improved stroke rates, shorter length of stay, and improved mortality as compared to an apical approach (10-12). For these reasons, we prefer a direct aortic strategy when necessary rather than an apical approach. However, the instances when this is necessary are rare. It is also important to recall that the TAVR trials for patients at low surgical risk mandated a transfemoral approach $(1,2)$. In low to intermediate risk patients SAVR should be considered if alternative access is necessary for TAVR. 

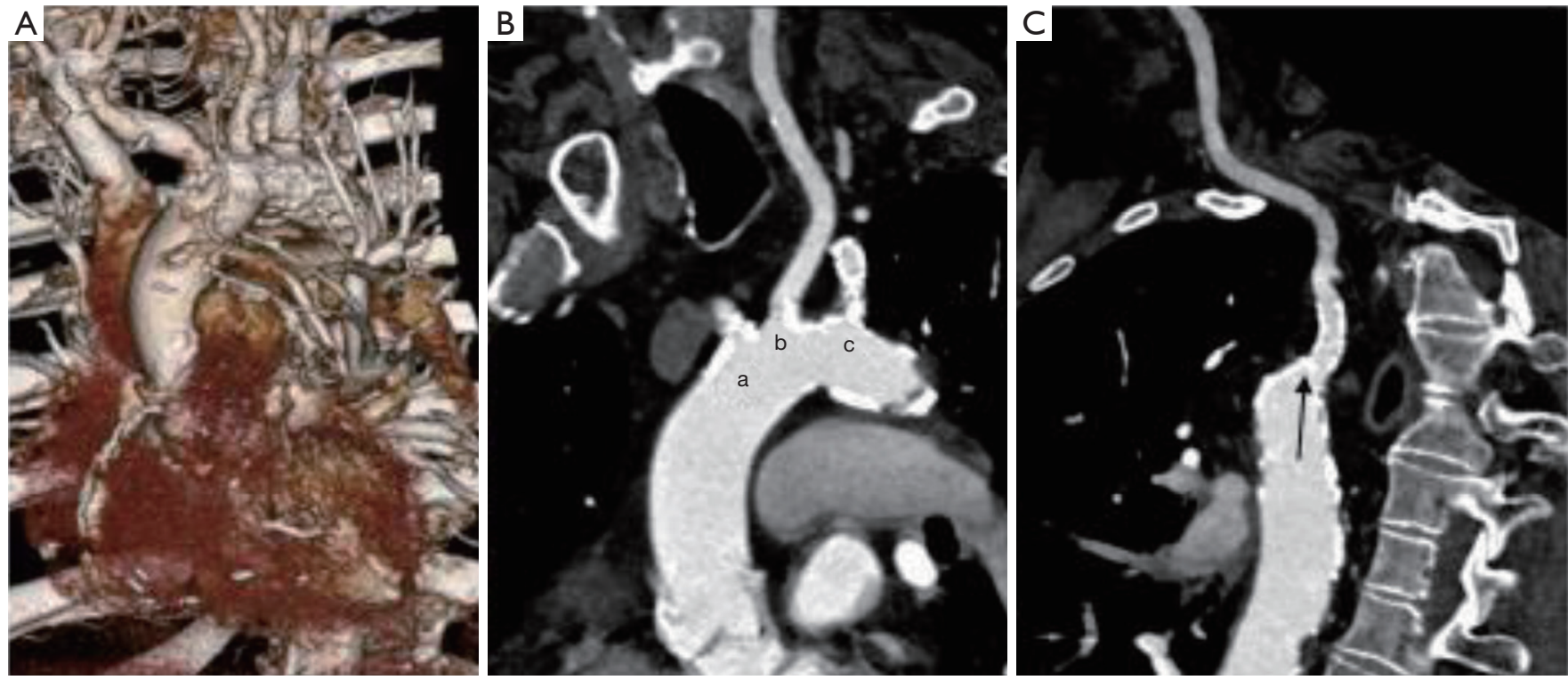

Figure 7 TAVR-CT in a patient that underwent direct aortic access. (A) 3D images from TeraRecon software demonstrating ascending aorta and aortic arch demonstrating appropriate landing zone above saphenous vein graft; (B) TAVR-CT images demonstrating calcified ostial stenosis of right brachiocephalic (a), left common carotid (b), and left axillary (c); (C) TAVR-CT demonstrating left axillary artery (arrow) with ostial stent. Left axillary artery was of borderline MLD and avoided given a patent LIMA to the left anterior descending artery. TAVR, transcatheter aortic valve replacement; MLD, minimum lumen diameter; CT, computed tomography.

\section{Pre-procedural planning}

The gated TAVR-CT is carefully reviewed and there needs to be an appropriate non-calcified access zone in the anterior aspect of the ascending aorta. Of note, approximately $6 \mathrm{~cm}$ of distance from the aortic annulus to the intended aortic access site is necessary to safely perform transaortic TAVR. In addition, if saphenous vein grafts are present it is important to localize them on TAVR-CT and ensure they are adequately away from the planned access zone. Figure 7 shows a patient that had inadequate transfemoral access and on review had inadequate carotid and subclavian access. The patient had borderline left subclavian access, but given a patent LIMA graft this site was not chosen. In addition, the left and right carotid had significant calcified plaque at the origin. The 3 -dimensional images from the TAVR-CT demonstrated an area just proximal to the right brachiocephalic artery above the saphenous vein graft with an appropriate sheath placement target.

\section{Procedure}

As above after the patient is prepped and placed under general anesthesia by our cardiac anesthesia team, femoral arterial and venous access are obtained for the transvenous pacer and pigtail catheter. Once access is obtained a mini partial upper sternotomy is performed. The sternum can be divided to either the $3^{\text {rd }}$ or $4^{\text {th }}$ intercostal space where the sternum can be divided to the right side. We do not perform the suprasternal approach due to the potential risk of lack of control of the aortic access site. Only a small area of disease-free ascending aorta is necessary to perform transaortic TAVR. If saphenous vein grafts are present, it is rarely an issue in the area of the aorta that is accessed. The transvenous pacing wire is then placed in the right ventricle and the pigtail catheter in the right coronary cusp via the femoral access.

Two pledgeted purse string sutures are then placed in the distal ascending aorta in the appropriate landing zone. The ascending aorta is directly accessed with a standard access needle via the mini upper sternotomy through the purse string sutures under fluoroscopic guidance and a 7-French sheath is placed in the ascending aorta. The aortic valve is crossed using an AL-1 catheter with a soft-tipped straight wire and then exchanged for a J-tip wire which is positioned in the left ventricular. A pigtail catheter is passed over the 
J-tip wire into the left ventricle and the J-tip is exchanged for a working wire. The 7-French sheath is then carefully exchanged for the sheath that was chosen based on the valve type. As the valve is introduced into the delivery sheath, it is carefully de-aired. The valve is then deployed similar to a transfemoral approach.

After valve deployment and confirmation of appropriate placement the catheters are removed over a wire. The introducer system for the device is removed from the access sheath. A period of rapid ventricular pacing is initiated to briefly minimize cardiac output. During the rapid pacing maneuver, the sheath is pulled out of the ascending aorta and the purse string sutures are tied with hemostasis.

\section{Transcaval access}

Transcaval TAVR is a relatively new approach that is the preferred alternative access strategy at select heart centers. During transcaval TAVR, an electrified guidewire is inserted through the inferior vena cava into an aortic snare that is inserted into the abdominal aorta. The sheath can then be advanced over the guidewire and through the inferior vena cava into the abdominal aorta and the procedure can be completed through this access. At the end of the case a nitinol occluder device is used to close the defect between the inferior vena cava and the abdominal aorta. Given that the pressure in the inferior vena cava should be lower than pressure in the retroperitoneum, blood exiting the abdominal aorta should preferentially enter the hole in the inferior vena cava during the procedure (17). A single arm multi-center study of 100 patients demonstrated high procedural success with few complications. However, the rate of life-threatening and major bleeding was high in this study at $13 \%$ and $7 \%$, respectively. This may be overestimated given that patients routinely had abdominal computed tomography post-procedure to identify retroperitoneal hemorrhage (17). Given our heart center's expertise and success with axillary and carotid access we have not adopted this approach and have not turned any patient away for lack of vascular access. Further studies comparing transcaval to axillary or carotid access should be undertaken before broad adoption of this technique.

\section{Apical access}

Transapical TAVR has consistently been associated with increased stroke rates and mortality and is primarily of historical significance at this time. During the early years of TAVR, transapical TAVR was the only option for an alternative access approach. In addition, initial sheath sizes were large and often precluded a transfemoral approach. As a result, Transapical TAVR was more common. In the initial PARTNER trial published in 2011, the sheath sizes were 22 -French or 24 -French and $30 \%$ of patients undergoing TAVR required apical access (18). As sheath sizes and delivery systems have improved new alternative access strategies have been introduced and apical access has become rare in the United States. In our program, using the algorithm described we have not performed a transapical TAVR since 2015 and have not turned any patients down for access related issues. Carotid and axillary access are less invasive and have been shown to have lower mortality and decreased length of hospitalization as compared to a direct aortic or apical approach. These strategies should be explored before proceeding with an apical approach. Several studies have shown trends that were not statistically significant, toward decreased mortality, lengths of hospitalization, and stroke rates with direct aortic access as compared to apical access $(10,12)$. Apical access should only be performed in extreme risk patients that do not have other surgical options. In addition, these patients should be referred to centers with expertise in transcarotid or transaxillary experience so these routes can be considered.

\section{Conclusions}

TAVR has become the most prevalent treatment strategy for patients with severe aortic stenosis and currently transfemoral access is clearly the preferred vascular access strategy. Most patients can safely be treated via a transfemoral approach. However, there is a significant burden of peripheral arterial disease, particularly in high risk patients, and transfemoral TAVR is not possible in some of these patients. However, as sheaths have become smaller a transfemoral approach is possible in greater than 90 percent of patients undergoing TAVR in the Unites States. TAVR-CTs should be routinely performed with imaging of the vascular anatomy from below the femoral head to mid-neck to completely evaluate femoral, aortic, carotid, and subclavian anatomy. This should be reviewed by the multidisciplinary heart team including cardiology, cardiothoracic surgery, and radiology to determine the preferred access strategy prior to the case.

There are currently no randomized data to support one alternative access strategy over another so the strategy should be chosen by the heart team based on patient 
anatomy, risk factors, and your center's expertise. Our heart center's algorithm prefers left transaxillary as our primary alternative access followed by transcarotid and then direct aortic if the patient's anatomy precludes axillary or carotid access. However, retrospective data suggest there may be an increased risk of stroke in the axillary approach which warrants further investigation. The vast majority of cases can be accomplished by alternative access via the axillary or carotid arteries and transapical access should be mostly of historical significance at this time in TAVR. Some TAVR centers have performed peripheral vascular interventions, including intravascular lithotripsy, in the femoral and iliac vasculature prior to TAVR in patients with inadequate femoral access to decrease the need for alternative access. There are currently no data to support the use of peripheral vascular interventions to obtain femoral access versus pursuing an alternative access strategy in a carotid or axillary artery with minimal atherosclerosis. Our institution has experienced excellent outcomes with the utilization of carotid and axillary access for TAVR in the minority (approximately $10 \%$ of patients) that have inadequate femoral access and we favor this approach.

Alternative access cases are relatively rare and should be referred to high volume TAVR centers that have significant alternative access volume. At this time, low and intermediate risk patients should be considered for SAVR if alternative access is necessary. As TAVR continues to progress we need outcome data comparing a carotid versus axillary approach to define the optimal alternative access strategy.

\section{Acknowledgments}

None.

\section{Footnote}

Conflicts of Interest: The authors have no conflicts of interest to declare.

Ethical Statement: The authors are accountable for all aspects of the work in ensuring that questions related to the accuracy or integrity of any part of the work are appropriately investigated and resolved.

\section{References}

1. Popma JJ, Deeb GM, Yakubov SJ, et al. Transcatheter Aortic-Valve Replacement with a Self-Expanding Valve in
Low-Risk Patients. N Engl J Med 2019;380:1706-15.

2. Mack MJ, Leon MB, Thourani VH, et al. Transcatheter Aortic-Valve Replacement with a Balloon-Expandable Valve in Low-Risk Patients. N Engl J Med 2019;380:1695-705.

3. Kundi H, Strom JB, Valsdottir LR, et al. Trends in Isolated Surgical Aortic Valve Replacement According to HospitalBased Transcatheter Aortic Valve Replacement Volumes. JACC Cardiovasc Interv 2018;11:2148-56.

4. Vemulapalli S, Carroll JD, Mack MJ, et al. Procedural Volume and Outcomes for Transcatheter Aortic-Valve Replacement. N Engl J Med 2019;380:2541-50.

5. Adams DH, Popma JJ, Reardon MJ, et al. Transcatheter Aortic-Valve Replacement with a Self-Expanding Prosthesis. N Engl J Med 2014;370:1790-8.

6. Reardon MJ, Van Mieghem NM, Popma JJ, et al. Surgical or Transcatheter Aortic-Valve Replacement in Intermediate-Risk Patients. N Engl J Med 2017;376:1321-31.

7. Smith CR, Leon MB, Mack MJ, et al. Transcatheter versus Surgical Aortic-Valve Replacement in High-Risk Patients. N Engl J Med 2011;364:2187-98.

8. Leon MB, Smith CR, Mack MJ, et al. Transcatheter or Surgical Aortic-Valve Replacement in Intermediate-Risk Patients. N Engl J Med 2016;374:1609-20.

9. Fanaroff AC, Manandhar P, Holmes DR, et al. Peripheral Artery Disease and Transcatheter Aortic Valve Replacement Outcomes: A Report From the Society of Thoracic Surgeons/American College of Cardiology Transcatheter Therapy Registry. Circ Cardiovasc Interv 2017. doi: 10.1161/ CIRCINTERVENTIONS.117.005456.

10. Allen K, Chhatriwalla A, Cohen D, et al. Transcarotid Versus Transapical and Transaortic Access for Transcatheter Aortic Valve Replacement. Ann Thorac Surg 2019;108:715-22.

11. Blackstone EH, Suri RM, Rajeswaran J, et al. Propensitymatched comparisons of clinical outcomes after transapical or transfemoral transcatheter aortic valve replacement: a placement of aortic transcatheter valves (PARTNER)-I trial substudy. Circulation 2015;131:1989-2000.

12. Chamandi C, Abi-Akar R, Rodes-Cabau J, et al. Transcarotid Compared With Other Alternative Access Routes for Transcatheter Aortic Valve Replacement. Circ Cardiovasc Interv 2018;11:e06388.

13. Dahle TG, Kaneko T, McCabe JM. Outcomes Following Subclavian and Axillary Artery Access for Transcatheter Aortic Valve Replacement: Society of the Thoracic 
Surgeons/American College of Cardiology TVT Registry

Report. JACC Cardiovasc Interv 2019;12:662-9.

14. Overtchouk P, Modine T. Alternate Access for TAVI: Stay Clear of the Chest. Interv Cardiol 2018;13:145-50.

15. Overtchouk P, Folliguet T, Pinaud F, et al. Transcarotid Approach for Transcatheter Aortic Valve Replacement With the Sapien 3 Prosthesis: A Multicenter French Registry. JACC Cardiovasc Interv 2019;12:413-9.

16. Schafer U, Deuschl F, Schofer N, et al. Safety and efficacy of the percutaneous transaxillary access for transcatheter aortic valve implantation using various transcatheter

Cite this article as: Banks A, Gaca J, Kiefer T. Review of alternative access in transcatheter aortic valve replacement. Cardiovasc Diagn Ther 2020;10(1):72-82. doi: 10.21037/ cdt.2019.10.01 heart valves in 100 consecutive patients. Int J Cardiol 2017;232:247-54.

17. Greenbaum AB, Babaliaros VC, Chen MY, et al. Transcaval Access and Closure for Transcatheter Aortic Valve Replacement: A Prospective Investigation. J Am Coll Cardiol 2017;69:511-21.

18. Leon MB, Smith CR, Mack M, et al. Transcatheter Aortic-Valve Implantation for Aortic Stenosis in Patients Who Cannot Undergo Surgery. N Engl J Med 2010;363:1597-607. 